\title{
RGD-mimic polyamidoamine-montmorillonite composites with tunable stiffness as scaffolds for bone tissue-engineering applications
}

\author{
Nicolò Mauro $^{1 \dagger *}$, Federica Chiellini ${ }^{2}$, Cristina Bartoli ${ }^{2}$, Matteo Gazzarri ${ }^{2}$, Michele Laus ${ }^{3}$, \\ Diego Antonioli ${ }^{3}$, Peter Griffiths ${ }^{4}$, Amedea Manfredi ${ }^{1}$, Elisabetta Ranucci ${ }^{1}$ and Paolo Ferruti ${ }^{1}$ \\ ${ }^{1}$ Dipartimento di Chimica, Università degli Studi di Milano, Italy \\ ${ }^{2}$ Dipartimento di Chimica e Chimica Industriale, Pisa, Italy \\ ${ }^{3}$ Dipartimento di Scienze ed Innovazione Tecnologica, Università del Piemonte Orientale 'A. Avogadro', Alessandria, Italy \\ ${ }^{4}$ Department of Pharmaceutical, Chemical and Environmental Sciences, Faculty of Engineering and Science, University of Greenwich, \\ Medway Campus, Kent, UK
}

\begin{abstract}
This paper reports on the development of montmorillonite (MMT)-reinforced hydrogels, based on a peptidomimetic polyamidoamine carrying guanidine pendants (AGMA1), as substrates for the osteoinduction of osteoblast precursor cells. AGMA1 hydrogels of various degrees of crosslinking responded favourably to MMT reinforcement, giving rise to composite hydrogels with shear storage modulus $G^{\prime}$, when fully swollen in water, up to $200 \mathrm{kPa}$, i.e. 20 times higher than the virgin hydrogels and of the same order or higher than other hydrogel-based composites proposed for orthopaedic applications. This significant improvement was ascribed to the effective interpenetration between the polymer matrix and the inorganic filler. AGMA1-MMT hydrogels, when evaluated as scaffolds for the osteogenic differentiation of mouse calvaria-derived pre-osteoblastic MC3T3-E1 cells, proved able to support cell adhesion and proliferation and clearly induced differentiation towards the osteoblastic phenotype, as indicated by different markers. In addition, AGMA1-MMT hydrogels proved completely degradable in aqueous media at $\mathrm{pH} 7.4$ and did not provide any evidence of cytotoxicity. The experimental evidence suggests that AGMA1-MMT composites definitely warrant potential as scaffolds for osteoblast culture and bone grafts.
\end{abstract}

\section{Introduction}

Scaffolds for hard tissue regeneration, besides being biocompatible, biodegradable to non-toxic products and capable of supporting cell adhesion and proliferation, must provide chemical and mechanical stimuli triggering

\footnotetext{
*Correspondence to: Nicolò Mauro, Dipartimento di Chimica, Università degli Studi di Milano, Via C. Golgi 19, 20133 Milano, Italy.E-mail: nicolo.mauro@unipa.it

Present address: Dipartimento di Scienze e Tecnologie
Biologiche Chimiche e Farmaceutiche, Laboratory of Blocompatible Polymers, Università Degli Studi di Palermo, Via Archirafi 32, 90123 Palermo, Italy.
}

osteoblastic cell response and bone formation (Tortelli and Cancedda, 2009; Hutmacher et al., 2007; Sellgren and $\mathrm{Ma}, 2012$ ). To this purpose, montmorillonite (MMT) nanocomposites with inorganic scaffolds, e.g. hydroxyapatite, have proved to be endowed with higher osteoinductivity than native hydroxyapatite (Charles-Harris et al., 2008). The osteo-inductive ability of MMT is also apparent in polymer-MMT nanocomposites, which usually exhibit higher osteo-inductivity compared with the corresponding native polymers. This effect has been largely attributed to the higher stiffness and mechanical strength imparted by MMT (Ray and Okamoto, 2003; Biswas and Ray, 2001; Giannelis, 1998). MMT nanocomposites with biodegradable polymers were found suitable for in vivo implantation owing to the bioeliminability of MMT, accompanied by antiseptic, 
antitoxic and mucostabilizing properties (Aguzzi et al., 2007; Huang et al., 2005; Kawasumi, 2004, Forni et al., 1989).

To obtain polymer/MMT nanocomposites, the polymer must intercalate within the MMT lamellae (Kawasumi, 2004; Chang et al., 2010). Several MMT-nanocomposites with hydrophilic biomaterials, e.g. chitosan (Paluszkiewicz et al., 2011; Joshi et al., 2012), poly( $N$-isopropylacrylamide) (Lee and Fu, 2003; Mujumdar and Siegel, 2008), poly(ethylene oxide) (Sharifi et al., 2012), poly(trimethylene carbonate)-poly(ethylene glycol) (Sharifi et al., 2012) and gelatin MMT (Haroun et al., 2009), were prepared in aqueous media. In these processes, water molecules favoured intercalation by penetrating the interlayer space, hence increasing interlamellar distance (Lee and Fu, 2003).

Polyamidoamines (PAAs) are well-known, hydrophilic, biodegradable and biocompatible polymers suited for several biotechnological applications (Ferruti, 2013). Crosslinked PAAs form hydrogels capable, in several instances, of promoting cell adhesion and proliferation (Ferruti et al., 2005a, 2005b; Emilitri et al., 2008; Jacchetti et al., 2008; Dos Reis et al., 2010; Mauro et al., 2012). Among these, hydrogels based on AGMA1, a guanidine-substituted peptidomimetic PAA designed to mimic RGD peptide (Figure 1), exhibited particularly good performances both in vitro and in vivo. In linear form, AGMA1 is water-soluble and contains three different ionizable groups/repeating unit: a strongly acidic carboxyl group $\left(p K_{\mathrm{a} 1}=2.3\right)$; a weakly basic tert-amine group $\left(p K_{\mathrm{a} 2}=7.4\right)$; and a strongly basic guanidine group $\left(p K_{\mathrm{a} 3}=12.1\right)$. It has isoelectric point 10.3 and at $\mathrm{pH} 7.4$ carries an average of 0.55 excess positive charges/unit (Franchini et al., 2006; Ranucci et al., 2009). AGMA1 hydrogels showed excellent biocompatibility and promoted adhesion and proliferation of many cell types, including neural cells, and have been used as tubular conduits for in vivo regeneration of the sciatic nerve in rats (Magnaghi et al., 2011). However, their mechanical properties are inadequate to support hard tissue regeneration. In a recent paper (Martello et al., 2014), AGMA1 hydrogels were successfully reinforced by co-polymerizing, vinyl-terminated AGMA1 oligomers with 2-hydroxyethylmethacrylate. A different approach is presented here. It was considered that AGMA1,

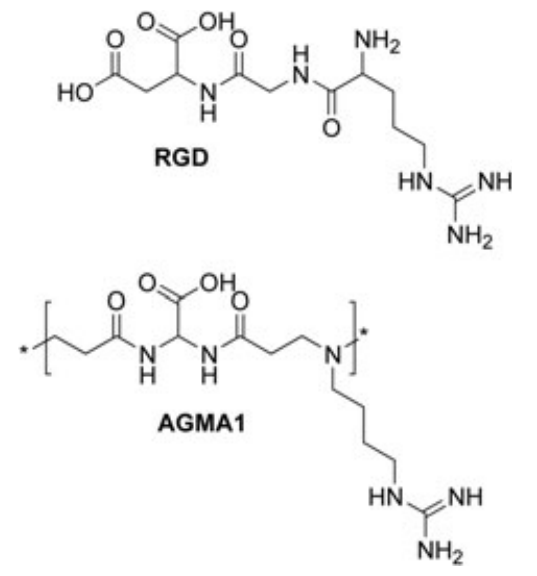

Figure 1. Representation of the structural similarities between the AGMA1 repeating unit and RGD tripeptide being prevailingly cationic, was particularly apt to interact with MMT platelets in aqueous media, establishing strong and complex polyelectrolytic bridges, which may act as additional physical crosslinkers. This would increase the stiffness of AGMA1 hydrogels without biasing their recognized cytobiocompatibility and cell-adhesive properties, and render them suitable scaffolds for hard tissue culture. This expectation was apparently fulfilled. The aim of this paper was to report on this issue.

\section{Materials and methods}

\subsection{Materials}

2,2-Bis(acrylamido-acetic acid (BAC; 95.5\%) was synthesized as previously described (Richardson et al., 1999). 1,2-Diaminoethane (EDA; 99.5\%), $\mathrm{Na}^{+}$MMT Nanoclay Nanomer® (cat. no. 682659, Sigma-Aldrich), ethanol (96\%), phosphate-buffered saline (PBS), pH 7.4, hydrochloric acid (37\%), 1-amino-4-guanidinobutane (agmatine) sulphate (97\%), lithium hydroxide monohydrate (98\%) and potassium chloride (99\%) were purchased from SigmaAldrich and used as received. $\alpha, \omega$-Acrylamide diterminated AGMA1 oligomers were prepared and characterized as previously described (Magnaghi et al., 2011), using the reagent amounts shown in Table 1.

\subsection{Mould preparation}

The moulds used for hydrogel preparation were two silanized $100 \times 100 \times 2 \mathrm{~mm}$ glass plates separated by a 2

Table 1. Reagent amounts used in the preparation of $\alpha, \omega$-acrylamide diterminated AGMA1 samples*

\begin{tabular}{lccc}
\hline Sample $^{\#}$ & Agmatine sulphate (mM) & BAC (mM) & LiOH- $\mathrm{H}_{2} \mathrm{O}(\mathrm{mm})$ \\
\hline AGMA10 & 17.4 & 19.3 & 36.7 \\
AGMA25 & 14.5 & 19.3 & 33.8 \\
AGMA30 & 13.5 & 19.3 & 32.8 \\
AGMA35 & 12.5 & 19.3 & 31.8 \\
AGMA40 & 11.6 & 19.3 & 30.9 \\
\hline
\end{tabular}

* In all preparations reagents were dissolved in $7 \mathrm{ml}$ water.

${ }^{\#}$ The number indicates the percentage of unbalanced double bonds in the preparation recipe.

Table 2. Reagents used in the preparation of AGMA1/MMT hydrogels

\begin{tabular}{lcccc}
\hline AGMA1-MMT & EDA (mg) & MMT $(\mathrm{mg})$ & Water $(\mathrm{ml})$ & Yield (\%) \\
\hline 1st series & & & & \\
AGMA10*/10 & 47.5 & 800.0 & 7.0 & 95.1 \\
AGMA30*/10\# & 182 & 800.0 & 7.0 & 95.3 \\
AGMA35*/10\# & 221 & 800.0 & 7.0 & 95.7 \\
AGMA40*/10 & 258 & 800.0 & 7.0 & 96.2 \\
2nd series & & & & \\
AGMA25*/0\# & 131.9 & - & 7.0 & 94.5 \\
AGMA25*/1 & 131.9 & 80.0 & 7.0 & 96.0 \\
AGMA25*/3 & 131.9 & 240.0 & 7.0 & 95.3 \\
AGMA25*/10\# & 131.9 & 800.0 & 7.0 & 92.9 \\
\hline
\end{tabular}

*The number indicates the degree of crosslinking (\%).

\# Percentage of MMT on a weight basis. 
mm-thick silicone spacer. Before use, the glass plates were soaked for $5 \mathrm{~h}$ in aqua regia, washed several times with water, dried and exposed for 3 days to chlorotrimethylsilane vapours in a closed chamber. They were then soaked with toluene $(20 \mathrm{ml})$, ethanol $(2 \times 20 \mathrm{ml})$ and water $(3 \times 20 \mathrm{ml})$, gently wiped and used immediately.

\subsection{AGMA1-MMT hydrogels}

(AGMA10/10 of Table 2 as model preparation) Bisacrylamide diterminated AGMA10 (8.0000 g, corresponding to $3.14 \mathrm{~mm}$ end-acrylamide groups) was dissolved in ultrapure water $(7.0 \mathrm{ml})$, the solution purged with nitrogen and MMT (0.8 g) very slowly added under stirring. The resultant homogeneous dispersion was stirred overnight under nitrogen, then EDA $(0.0475 \mathrm{~g}, 0.79 \mathrm{~mm})$ was added, the mixture warmed to $50^{\circ} \mathrm{C}$ and further stirred for $2 \mathrm{~h}$, then injected into the mould and kept for 5 days at $37^{\circ} \mathrm{C}$. The glass plates were then separated and the product recovered as an opalescent, tough and flexible sheet that was soaked for $24 \mathrm{~h}$ in ethanol $(200 \mathrm{ml})$ and for $4 \times 8 \mathrm{~h}$ in water $(200 \mathrm{ml})$.

\subsection{Swelling determinations}

Swelling experiments were performed in PBS, pH 7.4, on 2 $\times 12 \times 12 \mathrm{~mm}$ hydrogel discs previously dried to constant weight at 0.1 Torr. Each disc was placed in contact with the swelling medium ( $40 \mathrm{ml}$ ), kept for $24 \mathrm{~h}$ at $25^{\circ} \mathrm{C}$, retrieved and gently wiped with soft paper. The degree of swelling, $S W \%$, was determined by the following equation:

$$
S W \%=\frac{W_{0}-W_{t}}{W_{0}} \times 100
$$

where $W_{\mathrm{o}}$ is the weight of the dry disc and $W_{\mathrm{t}}$ is the weight of the swollen disc. Each experiment was repeated six times and the average values reported. The percentage variation coefficient, $C V \%$, was $<5 \%$.

\subsection{Size exclusion chromatography-low angle laser light scattering}

Size exclusion chromatography (SEC) traces were obtained with a Knauer Pump 1000 equipped with a Knauer Autosampler 3800, TSKgel G4000 PW and G3000 PW TosoHaas columns connected in series, a low-angle laser light scattering (LALS) Viscotek 270 Dual Detector, a UV detector (Waters Model 486) operating at $230 \mathrm{~nm}$, and a refractive index detector (Waters Model 2410). The mobile phase was a $0.1 \mathrm{M}$ Tris buffer, $\mathrm{pH} 8.00$ (0.05 with $0.2 \mathrm{M}$ sodium chloride). The flow rate was $1 \mathrm{ml} / \mathrm{min}$ and sample concentration $1 \% \mathrm{w} / \mathrm{w}$.

\subsection{Thermogravimetric analysis}

Thermogravimetric analyses were performed on a PerkinElmer TGA 4000 instrument equipped with an EGA furnace. Dried samples $(\approx 20 \mathrm{mg}$ ) were analysed in aluminium pans in an atmosphere of nitrogen flowing at 20 $\mathrm{ml} / \mathrm{min}$, at a heating rate of $10^{\circ} \mathrm{C} / \mathrm{min}$, from $30^{\circ} \mathrm{C}$ to $600^{\circ} \mathrm{C}$. The weight loss on heating of the dried composites was determined and reported as a function of time.

\subsection{FT-IR ATR spectroscopy}

FT-IR measurements were performed using a Spectrum 100 FT-IR spectrometer connected to a universal ATR sampling accessory (Perkin-Elmer) in the region 400-4000 $\mathrm{cm}^{-1}$. Spectra were measured at $3 \mathrm{~cm}^{-1}$ resolution. All samples were dried before performing measurements. Once the powdered solid sample had been placed on the diamond crystal area of the ATR accessory, the pressure was maintained at constant, pushing the sample onto the surface by means of a mechanical arm.

\section{8. $\zeta$-Potential analysis}

$\zeta$-Potential measures were performed using a Malvern NanoZS instrument. The electrophoretic mobility of the particles was evaluated for $2 \mathrm{~min}$ at $25^{\circ} \mathrm{C}$, applying a constant electric field, and $\zeta$-potential was calculated employing the Smoluchowski approximation. Each sample was analysed in triplicate, and the average $\zeta$-potential reported.

\subsection{Nanocomposite hydrogel degradation time}

The time required to solubilize the PAA matrix was considered the degradation time. It was determined with discs measuring $2 \times 10 \times 10 \mathrm{~mm}$, immersed in PBS, pH 7.4 (50 ml), and kept at $37^{\circ} \mathrm{C}$. Residual MMT was retrieved by centrifuging, extracting with $0.5 \mathrm{M}$ aqueous $\mathrm{NaCl}$ and then with water, and drying. The final solid was analysed by FT-IR and the degradation product characterized by SEC-LALS. All experiments were performed in triplicate with $\mathrm{CV} \% \leq 5 \%$.

\subsection{Rheological measurements}

Rheological tests were performed using an ARES straincontrolled rheometer (Rheometric Scientific); all tests were carried out at $25^{\circ} \mathrm{C}$, using parallel plate geometry ( $d=25 \mathrm{~mm}$ ). The linear viscoelastic zone was measured by applying a shear strain ramp of $0.05-100 \%$ at a frequency of $1 \mathrm{~Hz}$. The frequency sweep tests were performed at a strain of $0.1 \%$ over the frequency range $0.1-100 \mathrm{rad} / \mathrm{s}$. A compressive force of $1.0 \mathrm{~N}$ was applied to the hydrogels during the measurements. To prevent dehydration during rheological measurements, a thin layer of silicon oil was placed on the peripheral surface of the hydrogel held between the plates. Each measurement was performed at least twice on different disc specimens of the same hydrogel sample. 


\subsection{Cell culture and proliferation assays}

Mouse calvaria-derived pre-osteoblastic MC3T3-E1 cells from the American Type Culture Collection (ATCC; CRL 2594) were propagated using minimum essential medium, Eagle's $\alpha$-modification ( $\alpha$-MEM), containing ribonucleosides, deoxyribonucleosides, sodium bicarbonate and supplemented with $2 \mathrm{mM}$ L-glutamine, 1\% antibiotics (100 U/ml penicillium and $100 \mathrm{U} / \mathrm{ml}$ streptomycin), 10\% fetal bovine serum (FBS) and antimycotic, i.e. complete $\alpha$-MEM). Samples were cut, placed in 24-well culture plates and exposed to UV light for $1 \mathrm{~h}$. The samples were then covered with Dulbecco's phosphate-buffered saline (DPBS) with added penicillin-streptomycin solution (1\%) for $48 \mathrm{~h}$ and pre-incubated for an additional $3 \mathrm{~h}$ with complete $\alpha$-MEM. For all the biological investigations, the samples were seeded with MC3T3-E1 cells at a concentration of $1 \times 10^{5}$ and incubated at $37^{\circ} \mathrm{C}$ in a $5 \% \mathrm{CO}_{2}$-enriched atmosphere. The cells were allowed to proliferate for $24 \mathrm{~h}$ prior to incubation with osteogenic medium, prepared by adding $\gamma$-irradiated ascorbic acid $(50 \mu \mathrm{g} / \mathrm{ml})$ and $\beta$-glycerolphosphate disodium salt hydrate $(10 \mathrm{~mm}$ ) to the complete $\alpha$-MEM (Beck et al., 1998). Cells cultured on tissue-culture polystyrene (TCPS) were used as a control. Cell proliferation was investigated using WST-1 tetrazolium salt reagent (Roche) at days 3, 7, 14 and 21 after seeding. The cells were incubated for $4 \mathrm{~h}$ with WST- 1 reagent, diluted $1: 10$, at $37^{\circ} \mathrm{C}$ and $5 \% \mathrm{CO}_{2}$; formazan dye absorbance was measured, using a microplate reader, at $450 \mathrm{~nm}$, using $655 \mathrm{~nm}$ as reference.

\subsection{Alkaline phosphatase activity}

The alkaline phosphatase (ALP) activity of MC3T3-E1 cells cultured on AGMA1-MMT hydrogels was assessed at days 3, 7 and 14, using a colorimetric method based on the enzymatic conversion of $p$-nitrophenyl phosphate into $p$-nitrophenol. The hydrogels were washed three times with DPBS and then placed into $1 \mathrm{ml}$ lysis buffer, $\mathrm{pH} 10$, containing Triton X-100 (0.2\%), magnesium chloride $(5 \mathrm{~mm})$, trizma base $(10 \mathrm{~mm})$ and protease inhibitors cocktail $(2.5 \mu \mathrm{l} / \mathrm{ml})$. The samples were incubated at $4^{\circ} \mathrm{C}$ for $15 \mathrm{~min}$ and purified at $13000 \mathrm{rpm}$ for $10 \mathrm{~min}$. Afterwards, a solution containing glycine $(0.1 \mathrm{M})$, magnesium chloride (1 mM), zinc chloride (1 mM) and p-nitrophenyl phosphate $(1 \mathrm{mg} / \mathrm{ml})$ was added to $100 \mu \mathrm{l}$ sample and to pre-fixed concentrations of $p$-nitrophenol. The reaction was allowed to take place at $37^{\circ} \mathrm{C}$ for $30 \mathrm{~min}$ and then stopped by adding $50 \mu \mathrm{l} 2 \mathrm{M} \mathrm{NaOH}$ solution; the absorbance was measured at $405 \mathrm{~nm}$. ALP was normalized to the total protein content of each sample, which was measured using a BCA/protein assay (Ozkan et al., 2009). ALP activity was reported as $\mu \mathrm{M}$ product (mg protein $\times \mathrm{min})^{-1}$.

\subsection{Collagen production assessment}

The amount of collagen accumulated in the matrix following MC3T3-E1 cell culture was assessed at days 3, 7 and 14. Samples were washed with DPBS and the collagen content determined after digestion with pepsin dissolved in acetic acid $(0.5 \mathrm{M})$ for $16 \mathrm{~h}$ at $4^{\circ} \mathrm{C}$. Digested samples $(100 \mu \mathrm{l})$ were added to $1 \mathrm{ml}$ solution of Direct Red 80 $(50 \mu \mathrm{M})$ in acetic acid $(0.5 \mathrm{M})$ and incubated for $30 \mathrm{~min}$ at room temperature on an orbital shaker. The samples were then centrifuged at $13000 \mathrm{rpm}$ for $60 \mathrm{~min}$ at $4^{\circ} \mathrm{C}$, the supernatants drained and the pellets eluted with $1 \mathrm{~N}$ $\mathrm{NaOH}$ for $15 \mathrm{~min}$ at room temperature (RT). The supernatants $(100 \mu \mathrm{l})$ were transferred to 96 -well plates and their absorbance was measured at $540 \mathrm{~nm}$ (Ozkan et al., 2009; Beck et al., 1998; Richardson et al., 1999; Martello et al., 2014; Magnaghi et al., 2011; Franchini et al., 2006; Ranucci et al., 2009; Ferruti et al., 2005a, 2005b•; Emilitri et al., 2008; Jacchetti et al., 2008; Dos Reis et al., 2010; Mauro et al., 2012, Iyer et al., 2012), using a calibration curve obtained with collagen type I from calf's skin (Sigma, Milan, Italy). For comparison purposes, collagen accumulated by cells grown on TCPS under the same culture conditions was determined. Collagen amount was normalized to the total protein content of each sample. Unseeded hydrogels were used as blanks.

\subsection{Mineralized matrix deposition analysis by alizarin red staining (ARS)}

The mineralized matrix deposition was analysed by using the ARS method (Ozkan et al., 2009; Stanford et al., 1995; Nishikawa et al., 2012) after 14 and 21 days of cell culture. Hydrogels were fixed with $3.8 \%$ paraformaldehyde at RT for $30 \mathrm{~min}$, then stained with $2 \%$ alizarin red solution, $\mathrm{pH} \mathrm{4}$, for $10 \mathrm{~min}$. After the dye incubation, the samples were extensively rinsed with sterile deionized water in order to remove excess dye. To quantify the amount of calcium deposited on the hydrogels, the red matrix precipitate was dissolved in 10\% cetylpyridinium chloride (CPC) solution, $\mathrm{pH} 7$, and the optical density of the solution was read using a microplate reader at $565 \mathrm{~nm}$. Unseeded hydrogels were used as blanks.

\subsection{Confocal laser scanning microscopy (CLSM)}

The morphology of MC3T3-E1 cells cultured on the samples was investigated by means of CLSM at days 3, 7, 14 and 21. Cells were fixed with 3.8\% paraformaldehyde for $1 \mathrm{~h}$ in $1 \times \mathrm{PBS}$ at RT and permeabilized with a $1 \times$ PBSTriton X-100 solution (0.1\%) for $5 \mathrm{~min}$. After blocking with $1 \% \mathrm{w} / \mathrm{v}$ bovine serum albumin (BSA) in $1 \times$ PBS for 30 min, the cells were incubated with phalloidin (fluorescein isothiocyanate-labelled) in 1\% BSA solution at RT in the dark. After $30 \mathrm{~min}$, the cells were incubated with 4',6diamidino-2-phenylindole (DAPI) solution for $5 \mathrm{~min}$. Following dyeing incubation, the samples were extensively washed with $1 \times$ PBS before mounting on a glass slide for microscopic observation. A Nikon Eclipse TE2000 inverted microscope was used, equipped with an EZ-C1 confocal laser (Nikon, Japan) and a differential interference contrast 
(DIC) apparatus with $\times 20$ and $\times 60$ oil-immersion objectives. A $405 \mathrm{~nm}$ laser diode (405 nm emission) and an Argon ion laser (488 $\mathrm{nm}$ emission) were used to excite the DAPI and FITC fluorophores, respectively. Images were taken using Nikon EZ-C1 software, with identical settings for each sample, and further processed with GIMP (GNU Free Software Foundation) image manipulation software and merged using Nikon ACT-2U software.

\subsection{Statistical analysis}

The in vitro biological tests were performed in triplicate on each material. Data are represented as mean \pm standard deviation (SD). Statistical differences were analysed using one-way analysis of variance (ANOVA) and * $p<0.05$ and $* * p<0.001$ were considered significant.

\section{Results and discussion}

\subsection{Preliminary chemical considerations}

The aim of the present study was to assess the suitability of AGMA1-MMT composites as scaffolds for osteoblast culture and bone repair. AGMA1 is the poly-addition product of agmatine with 2,2-bis(acrylamido)acetic acid (BAC), whose repeating unit can be described as a RGD-like structure expressing similar biological properties (Figure 1). As RGD tripeptide is implicated in integrin-mediated cell recognition, it might be expected that such structural analogies could be exploited so as to promote cell adhesion and proliferation by mimicking in vivo conditions.

In the past, crosslinked AGMA1 hydrogels were prepared following two strategies. The first consisted of partly substituting 1,2-diaminoethane (EDA) for agmatine in the polymerizing mixture; the hydrogels so obtained were soft and fragile. The second strategy consisted of radically polymerizing AGMA1 bis-acrylamide diterminated oligomers prepared with monomer mixtures containing excess bisacrylamide; the resultant hydrogels were strong enough to prepare conduits for peripheral nerve regeneration in rats, but still too soft to be used for osteoblast culture and, eventually, for bone repair studies. The MMT-reinforced hydrogels reported in this paper were prepared in two steps (Scheme 1). In the first step, MMT was added to an aqueous solution of presynthesized bis-acrylamide di-terminated AGMA1 oligomers and the resultant mixture allowed to stand for $24 \mathrm{~h}$ at RT. The resultant slurry was then heated to $50^{\circ} \mathrm{C}$ and EDA added in 1:4 molar ratio to residual double bonds. The reaction mixture was maintained for $2 \mathrm{~h}$ at $50^{\circ} \mathrm{C}$ and then allowed to stand for 5 days at RT.

After repeated extractions with ethanol and ultrapure water, the AGMA1-MMT hydrogels were finally isolated as translucent films. The initial heating was necessary to ensure an even EDA diffusion inside the interlayers. The strategy adopted, of first preparing $\alpha, \omega$-acrylamide diterminated AGMA1 oligomers (coded as AGMA10, AGMA25, AGMA30, AGMA 35 and AGMA40; see Table 1) and then adding
MMT, and finally crosslinking the resultant AGMA1-MMT aggregates with EDA, had the additional merit of giving structured composites with predetermined average distances between two crosslink points.

\subsection{AGMA1-MMT interaction studies}

As a preliminary step, the ability of linear AGMA1 to interact with MMT was assessed. In particular, experiments were carried out in order to establish the ability of MMT and AGMA10, taken as a typical example, to form aggregates in aqueous media. A suspension of MMT (5 mg/ml) was stirred in the presence of AGMA10 (1:1 w/w) at $\mathrm{pH}$ 8.5. Aggregation phenomena obviously occurred. The aggregates were retrieved by centrifuging and the particles resuspended in water and recentrifuged several times to extract soluble fractions, if present. (DLS) analyses showed larger average diameter and lower polydispersity index $(P d I)$ for the AGMA10/MMT aggregates (diameter $5 \mu \mathrm{m}, P d I$ 0.27) than the native MMT (diameter $366 \mathrm{~nm}, P d I$ 0.64), suggesting effective electrostatic interactions among the AGMA1 matrix and MMT, probably deriving from both intercalation and flocculation phenomena. AGMA10-MMT particles were characterized by FT-IR spectroscopy and the absorption spectrum compared with those of MMT and linear AGMA10 (Figure 2).

The AGMA10-MMT spectrum showed several diagnostic bands typical of AGMA1 structure, e.g. those relative to C-H stretching (2960-2860 $\left.\mathrm{cm}^{-1}\right)$, asymmetric $\left(1619 \mathrm{~cm}^{-1}\right)$ and symmetric $\mathrm{COO}^{-}$stretching $\left(1379 \mathrm{~cm}^{-1}\right)$, as well as the amide I band $\left(1523 \mathrm{~cm}^{-1}\right)$. It also contained diagnostic bands of MMT, such as those relative to $\mathrm{O}-\mathrm{H}$ stretching $\left(3610 \mathrm{~cm}^{-1}\right)$ and $\mathrm{Si}-\mathrm{O}$ stretching $\left(1104 \mathrm{~cm}^{-1}\right)$, indicating that the product was indeed an MMT and AGMA10 combination.

Moreover, changes in the relative intensities of the bands in the $1100-800 \mathrm{~cm}^{-1}$ range were observed by comparing the spectra of the composite with those of parent MMT and AGMA10. In particular, the change of the relative intensities of the bands characteristic of bending vibrations of $\mathrm{AlFeOH}, \mathrm{AlAlOH}$ and $\mathrm{AlMgOH}$ (971, 913 and $841 \mathrm{~cm}^{-1}$, respectively) and the band characteristic of Si-O stretching $\left(1104 \mathrm{~cm}^{-1}\right)$ were clear signs of strong interactions between AGMA10 and $\mathrm{Si}-\mathrm{O}$ groups (Ray and Okamoto, 2003). At pH 8.5, the side guanidine groups of AGMA1 were completely protonated, whereas its chain tert-amine groups were $<10 \%$ protonated and the side carboxyl groups completely deprotonated (Ranucci E et al., 2009). It is therefore highly probable that electrostatic interactions were established among the PAA guanidine groups and the interlayer anionic groups of MMT. According to this hypothesis, the increasing polarizing power of the interlayer cation (guanidinium $>>\mathrm{Na}^{+}$) reflects on the decrement of the position and the intensity of the broad band near $3400 \mathrm{~cm}^{-1}$ attributed to the stretching H-O-H vibration.(Madejovà, 2003).

$\zeta$-Potential analyses of AGMA10/MMT particles revealed a more anionic and narrower surface charge distribution than that of parent MMT, with $\zeta$-potential values 

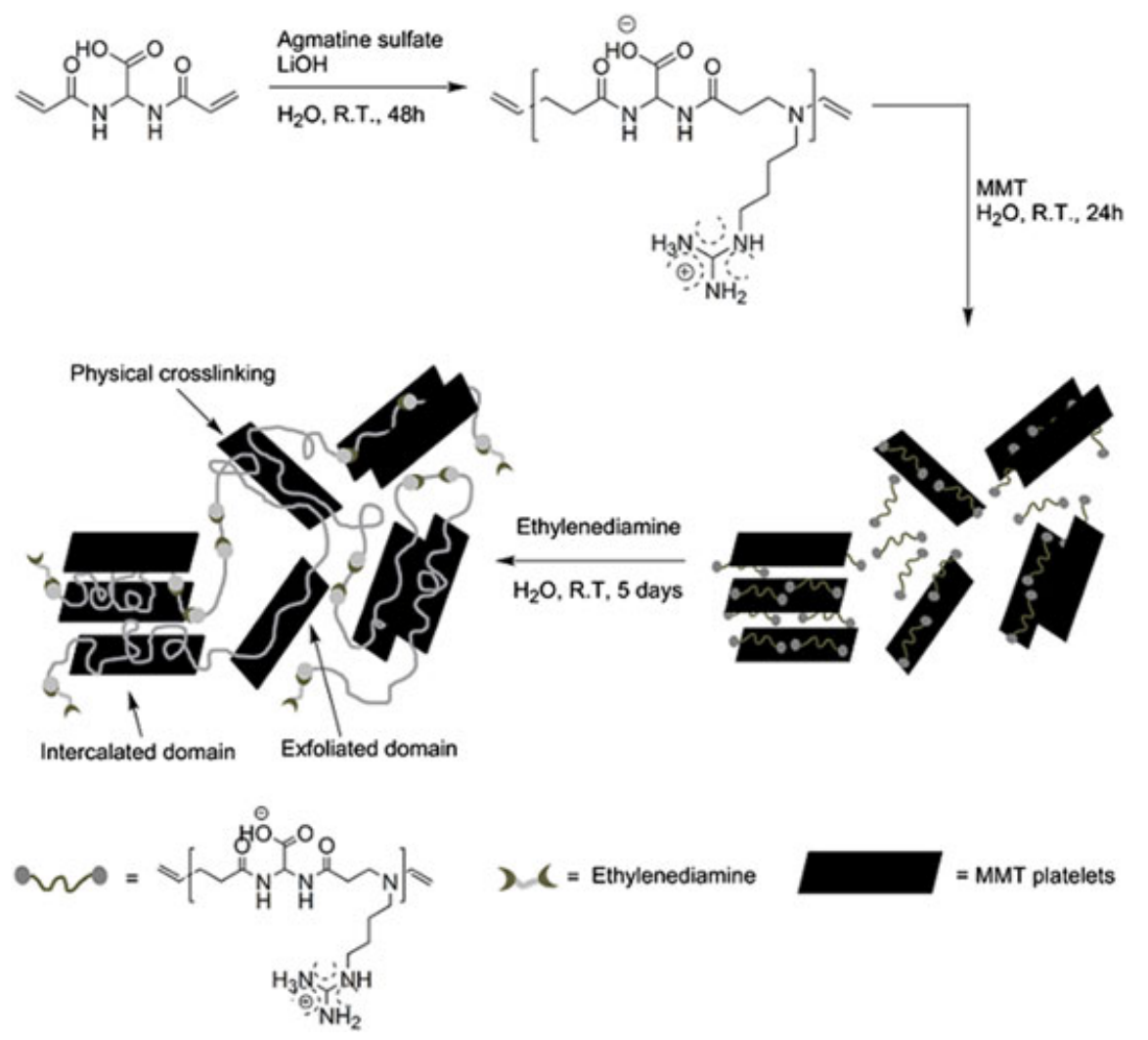

Scheme 1. Synthesis of AGMA1-hydrogel composites.

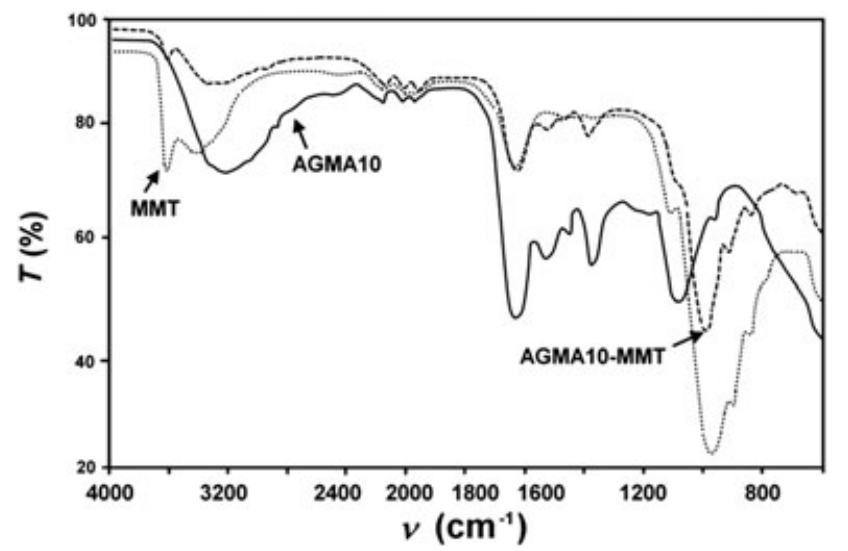

Figure 2. FT-IR spectra of AGMA1-MMT particles, raw MMT and AGMA1

of $-3 \pm 2.0 \mathrm{mV}$ for MMT and $-9.1 \pm 1.3 \mathrm{mV}$ for AGMA1MMT. It may be reasonably supposed that, as a consequence of the substitution of the sodium counterions in the interlayer channels by the cationic groups of AGMA1, the strongly acidic carboxyl groups, as well as the guanidine sulphate counterions present $a b$ initio, will form a negatively charged counterlayer on the outer particle surface, pointing to the aqueous phase.

\subsection{Hydrogel preparation and characterization}

Hydrogels were obtained as sheets $(100 \times 100 \times 2 \mathrm{~mm})$ and classified according to their degree of crosslinking and w\% MMT content, e.g. AGMA25/1 stands for the AGMA1-MMT nanocomposite with $25 \%$ degree of crosslinking and 1\% MTT, and so on (Table 1).

Two series of MMT-reinforced AGMA1 composite hydrogels were prepared. In the first series, the MMT content was maintained constant at $10 \%$, while varying the degree of crosslinking, according to the different lengths of the starting bis-acrylamide diterminated oligomers (Table 1 , first series). In the second series, the degree of crosslinking was constantly $25 \%$, whereas the MMT content varied in the range $1-10 \%$ (Table 1, second series, AGMA25/10 belonging to both series). The $25 \%$ degree of crosslinking was chosen for the AGMA1-MMT hydrogels of the second series because it combined good mechanical properties, with the preservation of a sufficient cationic character to interact strongly with varying amounts of MMT. In fact, the shorter the starting $\alpha, \omega$-acrylamide diterminated oligomer, the more its acid-base balance shifted towards the acidic side and was not entirely re-established by EDA, which introduced only two amine groups every four carboxylated terminals. The AGMA1-MMT hydrogels of the first series, having various degrees of crosslinking but constant (10\%) MMT content, were highly swollen by PBS buffer, $\mathrm{pH}$ 7.4. Rather surprisingly, with the exception of AGMA10/10, the degree of swelling increased by increasing the degree of crosslinking, passing from $75 \%$ for AGMA10/25 to $190 \%$ for AGMA35/10 and jumping to $370 \%$ for AGMA40/10 (Figure 3A). Two conflicting factors affected the degree of swelling. The increase of degree of crosslinking is normally expected to decrease swelling. However, at $\mathrm{pH} 7.4$, passing from the least to the 

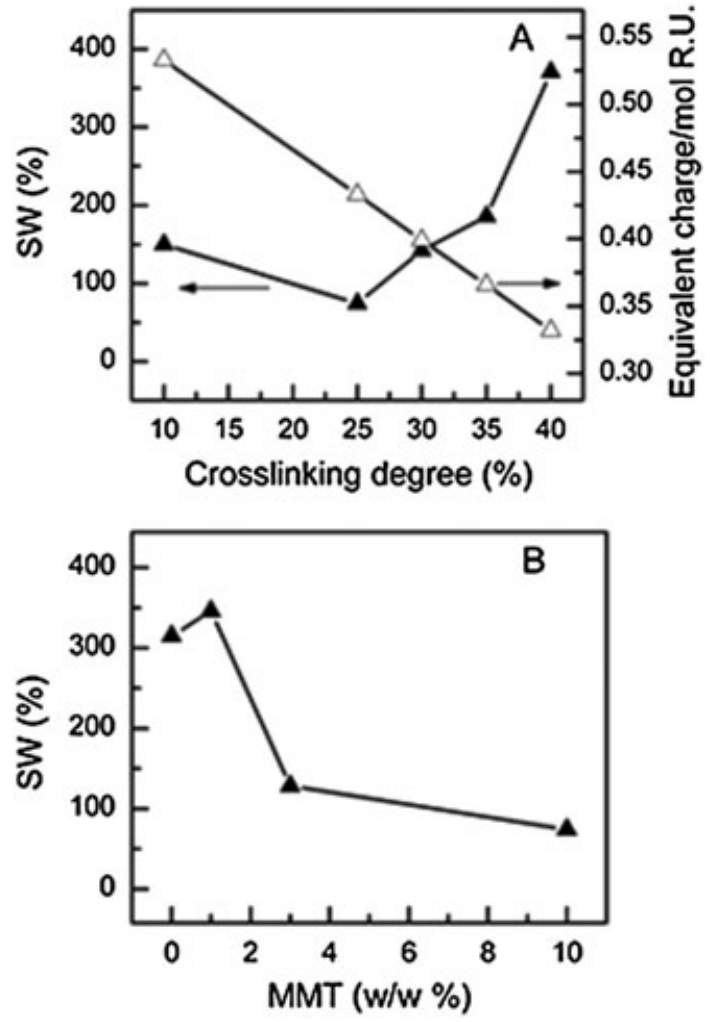

Figure 3. (A) Trend of the degree of swelling ( $\Delta$ ) and equivalent charge/mole of repeating units ( ) for AGMA1-MMT hydrogels with 10\% MMT and different degrees of crosslinking. (B) Trend of the degrees of swelling for AGMA25-MMT hydrogels

most crosslinked hydrogel, the net average charge passed from 0.52 to 0.32 positive charges/unit and the strength of AGMA1-MMT interactions decreased accordingly. This second factor loosened the composite network and apparently prevailed at a high degree of crosslinking (Figure 3A).

For the AGMA25-MMT series, the degree of swelling decreased from ca. $350 \%$ to $80 \%$ by increasing the MMT content from $1 \%$ to $10 \% \mathrm{w} / \mathrm{w}$ (Figure $3 \mathrm{~B}$ ). A deviation occurred for the AGMA25/1 hydrogel, whose degree of swelling was feebly higher than that of virgin AGMA25. This was ascribed to the expansion of the polymer network following the chain arrangements induced by the addition of a small amount of MMT. For all other AGMA-MMT hydrogels, the networks arising from the intercalation of increasing amounts of AGMA1 into the MMT sheets gave increasingly tight networks, with correspondingly decreasing aptitude to swell. Reinforcement of hydrogel matrices with MMT is known to induce an increase in thermal stability, as a consequence of the interactions among the clay platelets and polymer chains. This was confirmed in this study. The results of the TGA analysis of the AGMA25MMT series are reported in Figure 4. The virgin hydrogel showed a weight loss starting at temperatures slightly $>$ $100^{\circ} \mathrm{C}$, due to the release of hydration water, and proceeded with a nearly regular slope, with only minor inflections at $200^{\circ} \mathrm{C}$ and $400^{\circ} \mathrm{C}$. By contrast, the thermograms of all reinforced hydrogels showed stepwise degradation patterns. Moreover, confirming the general trend, all thermograms of the MMT-reinforced hydrogels

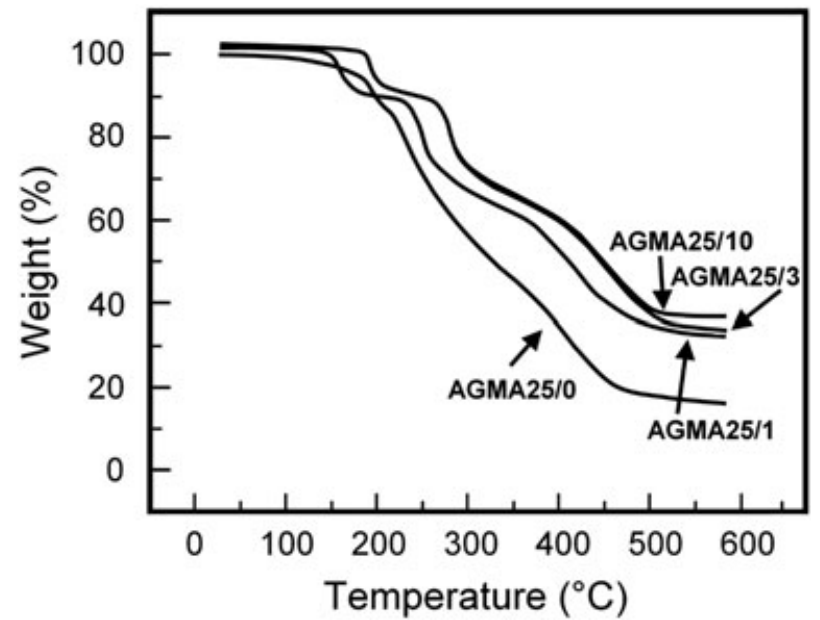

Figure 4. Thermograms of AGMA25-MMT hydrogels; heating rate, $20^{\circ} \mathrm{C} / \mathrm{min}$

were displaced towards higher temperatures compared to the virgin hydrogel, more so for those with higher MMT content. In addition, their degradation residues at $600^{\circ} \mathrm{C}$ were definitely much higher. The only exception was the portion of the AGMA25/1 hydrogel thermogram in the range $160-200^{\circ} \mathrm{C}$, which mostly remained below that of the virgin hydrogel. The two thermograms crossed slightly above $200^{\circ} \mathrm{C}$.

Degradation tests were carried out at $37^{\circ} \mathrm{C}$ in $\mathrm{PBS}, \mathrm{pH}$ 7.4. The MMT residue was exhaustively extracted with water, checking the absence of absorbed AGMA1 residues by FT-IR spectroscopy. The extracts were then lyophilized and the content analysed by SEC-LALS. No AGMA1 residues were detected with molecular weights $>1000$. The results are reported as a function of both the degree of crosslinking (Figure 5A) and the MMT content (Figure 5B). Broadly speaking, the degradation time of the hydrogels of the first series increased by increasing the degree of crosslinking to 25\% (AGMA25/10), then sharply decreased for AGMA40/10, probably as a consequence of its remarkably higher swelling. The degradation times of the hydrogels of the second series were in the range 45-144 days, the degradation time constantly increasing by increasing the MMT content.

\subsection{Rheological characterization}

The rheological properties of AGMA1-MMT hydrogels were determined in shear by means of strain and frequency sweep tests. Strain sweep tests were performed to define the linear viscoelastic zone. A compressive force of $1.0 \mathrm{~N}$ was applied, on account of the compressive force dependence of the storage and loss shear modulus (Mauro et al., 2012). Samples were deformed in a wide shear strain range, i.e. $0.05-100 \%$ at $1.0 \mathrm{~Hz}$. Typical strain test curves are reported in Figure 6 for AGMA25/0 and AGMA25/1.

For AGMA25/0, the shear storage modulus $G^{\prime}$ stayed constant up to $1 \%$ shear strains and then decreased for 


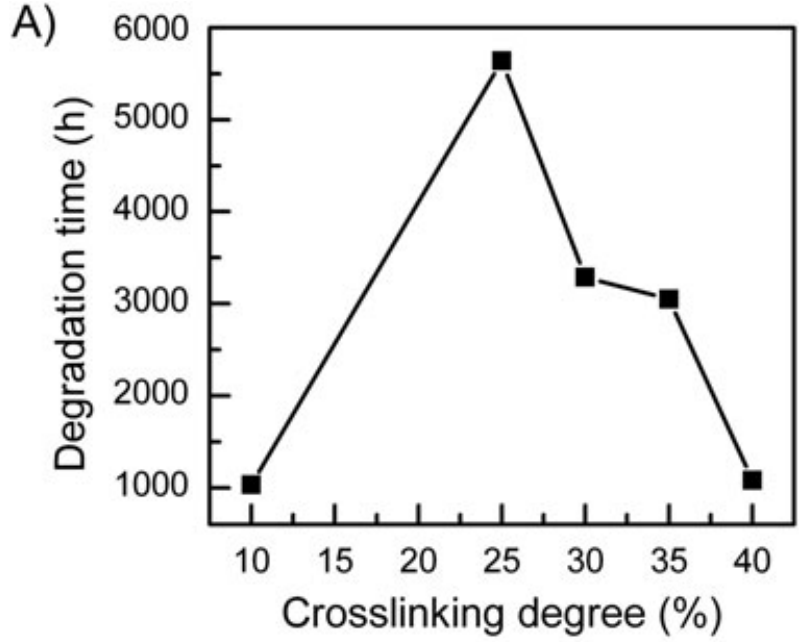

B)

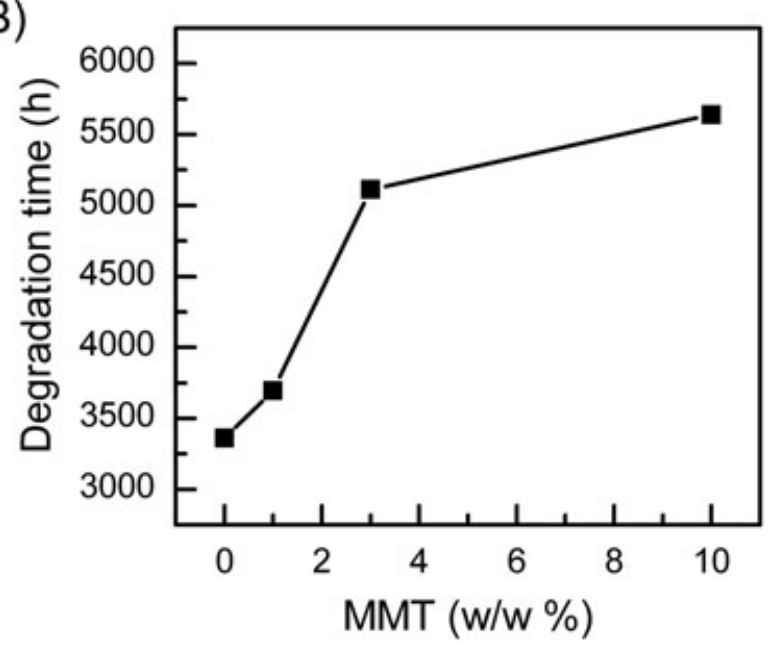

Figure 5. (A) Degradation time of AGMA1-MMT hydrogels with 10\% MMT and different degrees of crosslinking. (B) Degradation time of AGMA25-MMT hydrogels

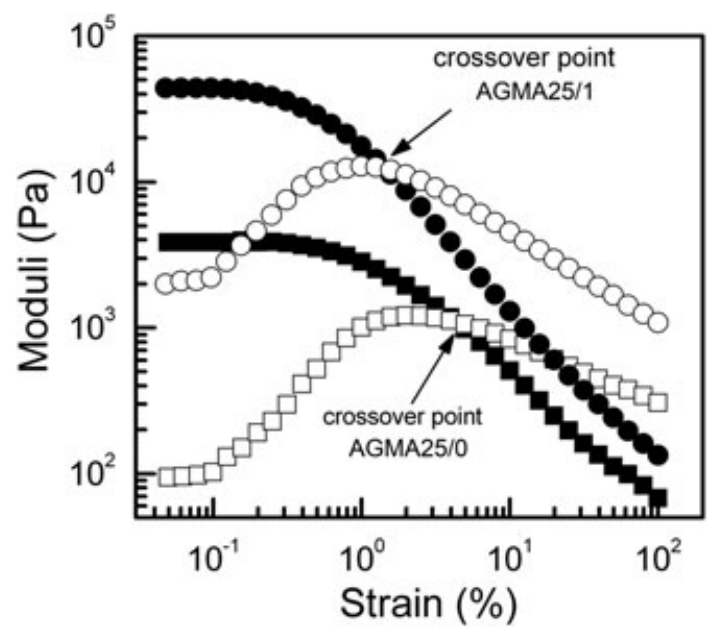

Figure 6. Strain sweep tests for AGMA25/0 and AGMA25/1: $G^{\prime}$, filled symbols; $G^{\prime \prime}$, open symbols

shear strains exceeding this critical value, indicating the transition from linear to non-linear behaviour (Ma et al., 2009). AGMA25/1 showed a reduction of the linear viscoelastic zone with respect to AGMA25/0. Frequency sweep tests were performed with a $0.1 \%$ deformation (linear viscoelastic range) over the frequency range 0.1-100 $\mathrm{rad} / \mathrm{s}$. The results obtained for hydrogels of the AGMA25-MMT series are reported in Figure 7. All samples showed a solid-like behaviour $\left(G^{\prime}>G^{\prime \prime}\right)$, with $G^{\prime}$ independent of the applied frequency, indicating the formation of well-developed crosslinked network (Lazzeri et al., 1994; Renò et al., 2013; Söderqvist Lindblad et al., 2005; Cascone et al., 1995).

Figure 8 reports the trends of $G^{\prime}$ at $100 \mathrm{rad} / \mathrm{s}$, obtained from the frequency sweep tests on samples with $10 \%$ MMT content Okay \& Oppermann (2007) as a function of the degree of crosslinking (Figure 8A) and with samples with $25 \%$ degree of crosslinking as a function of the MMT content (Figure 8B). $G^{\prime}$ increased up to 35\% degree of crosslinking (Mauro et al., 2012), collapsing for higher values (Figure 8A), probably due to the occurrence of two opposing factors. The AGMA1-MMT interactions decreased with increasing the degree of crosslinking as a consequence of the reduction of the overall positive charge (Figure $8 \mathrm{~A}$ ). At degrees of crosslinking $>35 \%$,
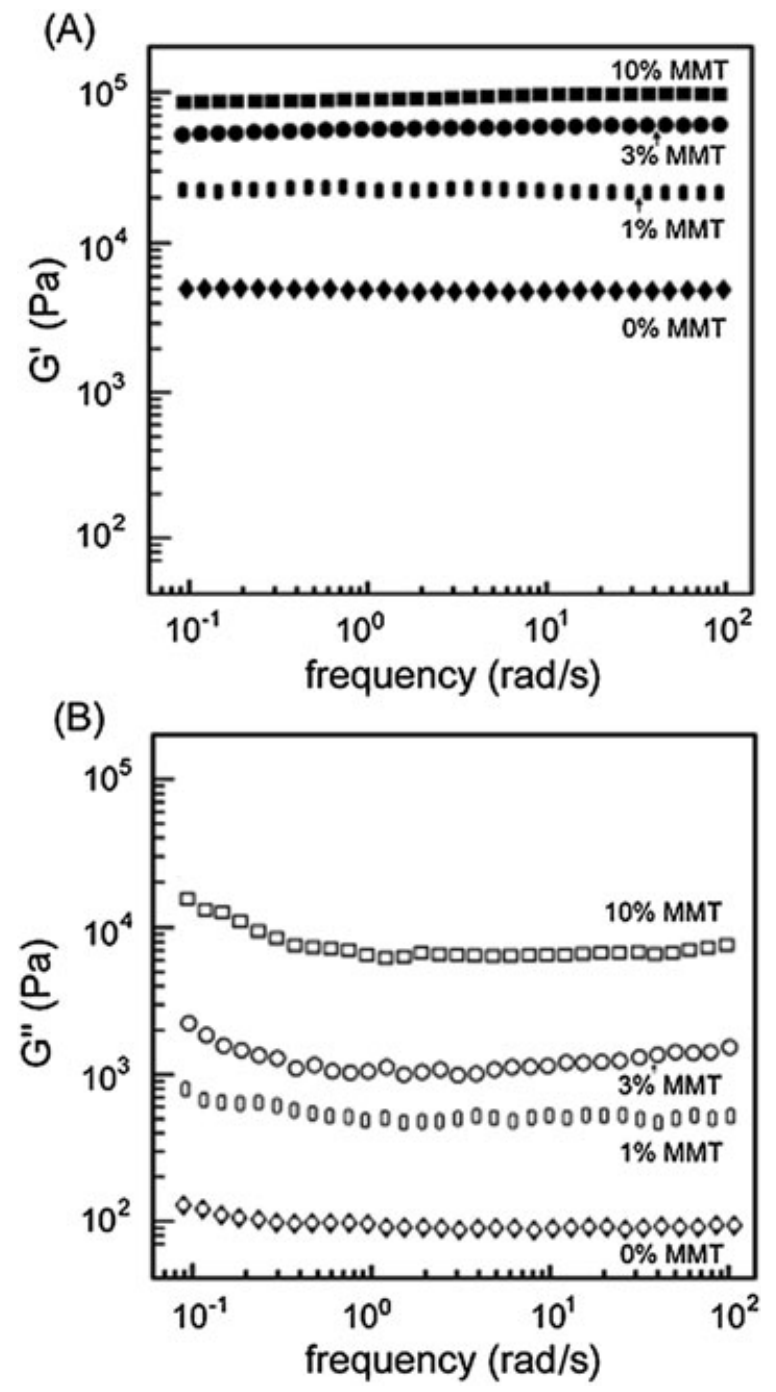

Figure 7. Frequency sweep test for AGMA25-MMT hydrogels: (A) $G^{\prime}$ and (B) $G^{\prime \prime}$ 


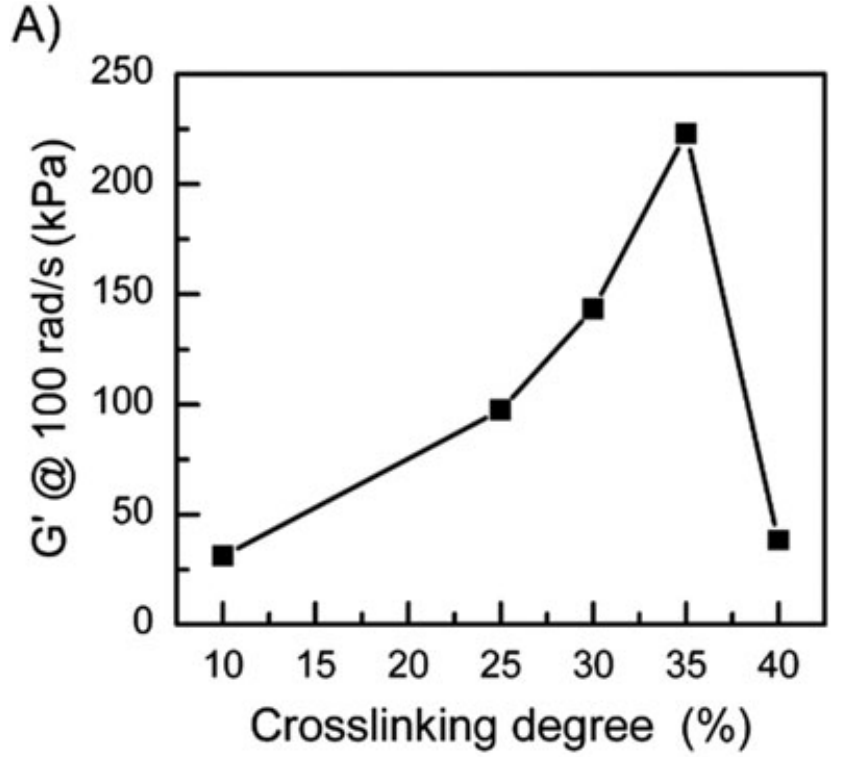

B)

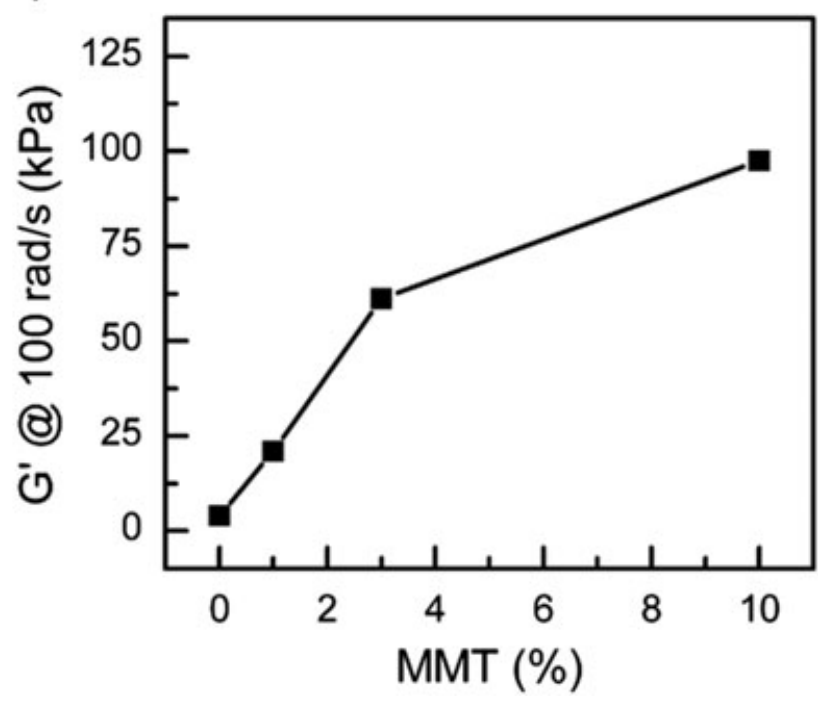

Figure 8. (A) Trend of the shear storage modulus $G^{\prime}$ at 100 rads $^{-1}$ (frequency sweep tests) for AGMA1-MMT hydrogels with $10 \%$ MMT and different degrees of crosslinking. (B) Trend of the shear storage modulus $G^{\prime}$ at $100 \mathrm{rads}^{-1}$ (frequency sweep tests) for AGMA25-MMT hydrogels

this effect counterbalanced the stiffening effect of crosslinking. Within the sample series with constant degree of crosslinking and variable MMT content (Figure 8B), $G^{\prime}$ increased with the MMT content (Cascone et al., 1995), more markedly so in the $1-3 \%$ range.

These data, together with the FT-IR ATR study reported above and the small-angle neutron scattering results (see Supplementary materials and methods, Figure S1), suggest that an efficient interpenetration of the polymer matrix with MMT platelets was accomplished, giving rise to complex and robust networks with tunable mechanical properties and stiffness until 20 times higher than those of common PAAs or other biomimetic matrices (i.e. $\approx 20$ $\mathrm{kPa}$ vs $220 \mathrm{kPa}$ ) (Paluszkiewicz et al., 2011; Joshi et al., 2012; Lee and Fu, 2003; Mujumdar and Siegel, 2008; Sharifi et al., 2012; Haroun et al., 2009).

\subsection{Biological studies}

Hydrogels of the AGMA25-MMT series with MMT amounts in the rang $0-10 \%$ were evaluated as scaffolds for cell culture, with potential for the osteoblastic differentiation of mouse calvaria-derived pre-osteoblastic (MC3T3-E1) cells. Cell proliferation was quantitatively evaluated by means of the WST- 1 assay at days 3, 7, 14 and 21 . The results reflected significant proliferation on all hydrogels after day 3 (Figure 9A), in agreement with the qualitative findings of CLSM analysis. Best proliferation values were observed for AGMA25/10, with highest $G^{\prime}$ modulus, highlighting that cell proliferation, migration and adhesion were strongly affected by the elastic response provided by the hydrogel scaffolds.

The progression towards the osteoblast phenotype of MC3T3-E1 cells was determined by monitoring ALP activity. ALP is a glycosylated membrane-bound enzyme catalysing the hydrolysis of phospho-mono-ester bonds and playing a physiological role in the metabolism of phospho-ethanolamine and inorganic pyrophosphate. The bone isoform of ALP is considered an early marker of osteoblastic phenotype expression. Interestingly, all AGMA25-MMT hydrogels, including the unreinforced AGMA25/0, showed a significantly higher ALP activity than the TCPS control at all time points (Figure 9B), with $p<0.05$ for AGMA25/0 and AGMA25/1 and $p<0.001$ for AGMA25/10, suggesting that the RGD-like domains of the AGMA1 matrix play a fundamental rule in addressing cell differentiation. A marked differentiation was observed after 3 days, with the highest value at day 14. ALP values were always maximal for AGMA25/10, significantly higher than that of AGMA25/0 ( $p<0.001)$, demonstrating the osteogenic rule of MMT on MC3T3 cells.

Collagen, a major extracellular matrix (ECM) component, is a good marker of osteoblast differentiation. Collagen accumulation onto AGMA25-MMT hydrogels following MC3T3-E1 culture was therefore quantitatively estimated at days 3, 7 and 14 of culture by the Direct Red 80 assay (Figure 9C). Whilst the amount of collagen produced on TCPS ( $p<0.001$ ) was modest, the hydrogels induced production of increasing amounts of collagen throughout the whole culture time, highest for AGMA25/10 at all time points. In particular, at day 14, collagen accumulation on AGMA25/10 $(p<0.001)$ was $\sim 80 \mu$ g, a significantly higher amount than in all other samples.

ECM mineralization represents a late-stage indicator of osteoblastic phenotype. ECM mineralization of MC3T3-E1 cells cultured on AGMA25-MMT hydrogels was quantitatively evaluated by alizarin red staining (ARS).

The amount of calcium-linked dye was measured at days 14 and 21 (Figure 9D). Significant levels of alizarin red dye were detected in all samples, significantly higher (differences $p<0.05$ ) than those obtained from cells grown on TCPS at all time points, with an increasing trend during the culture period. AGMA25/10 and, to a lesser extent AGMA25/1, showed the highest amount of dye at day 21 , with a remarkable difference compared to MMT-free AGMA25/0 ( $p<0.001)$. These results clearly 

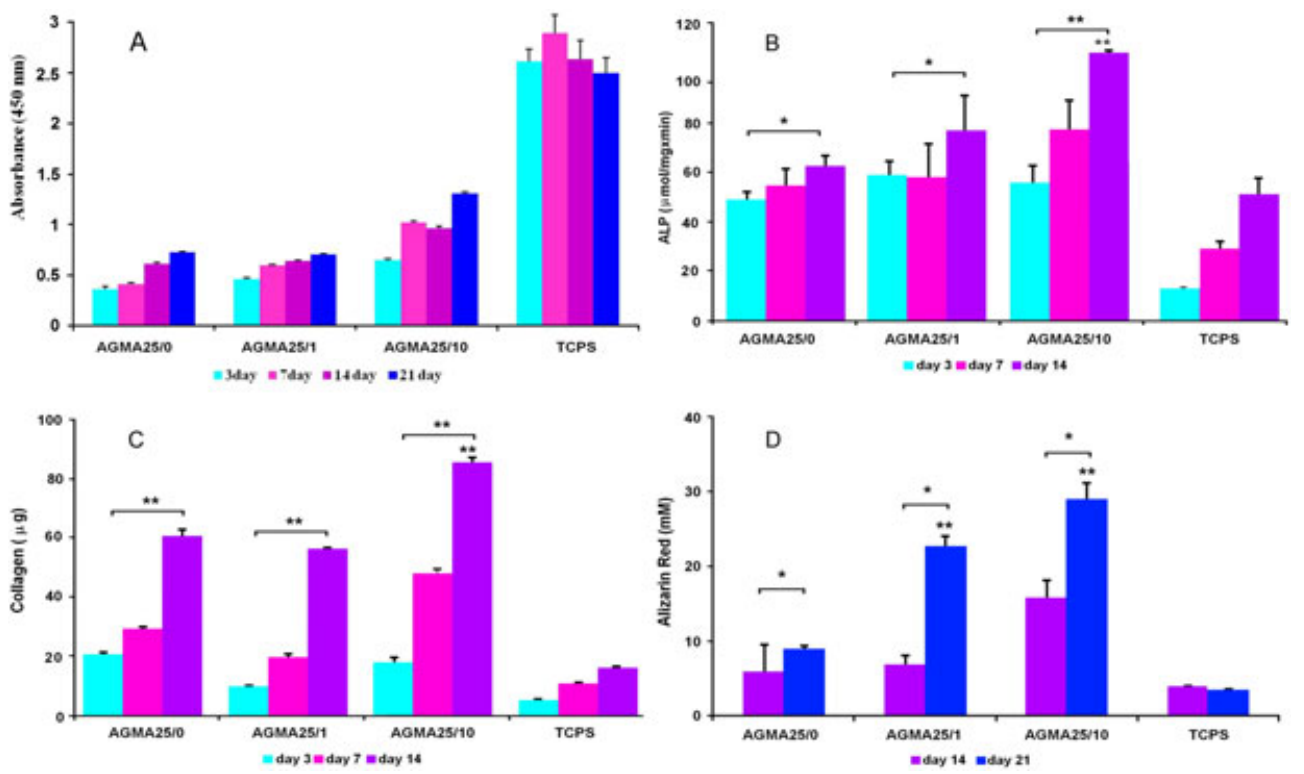

Figure 9. MC3T3-E1 culture on AGMA25-MMT hydrogels: (A) cell proliferation, evaluated by WST-1 assay; (B) ALP activity; (C) col-lagen production; (D) mineralization.

confirmed the capability of the MMT-containing hydrogels to better support the matrix mineralization process, obtaining high-performance biomaterials for potential in situ bone regeneration.

Qualitative analysis of cell morphology and cytoskeleton organization of MC3T3-E1 cells cultured on AGMA25MMT samples was obtained by means of CLSM. Cells were stained for F-actin with FITC-phalloidin and nuclei with DAPI. Adherent cells were clearly visible from day 3 until day 21 (Figure 10). The cells were well interconnected and formed crowded networks on all hydrogels.

Higher magnifications $(\times 60)$ revealed undifferentiated cell populations with a fusiform appearance, as well as cells with polygonal and star-shaped (osteocytes) morphology

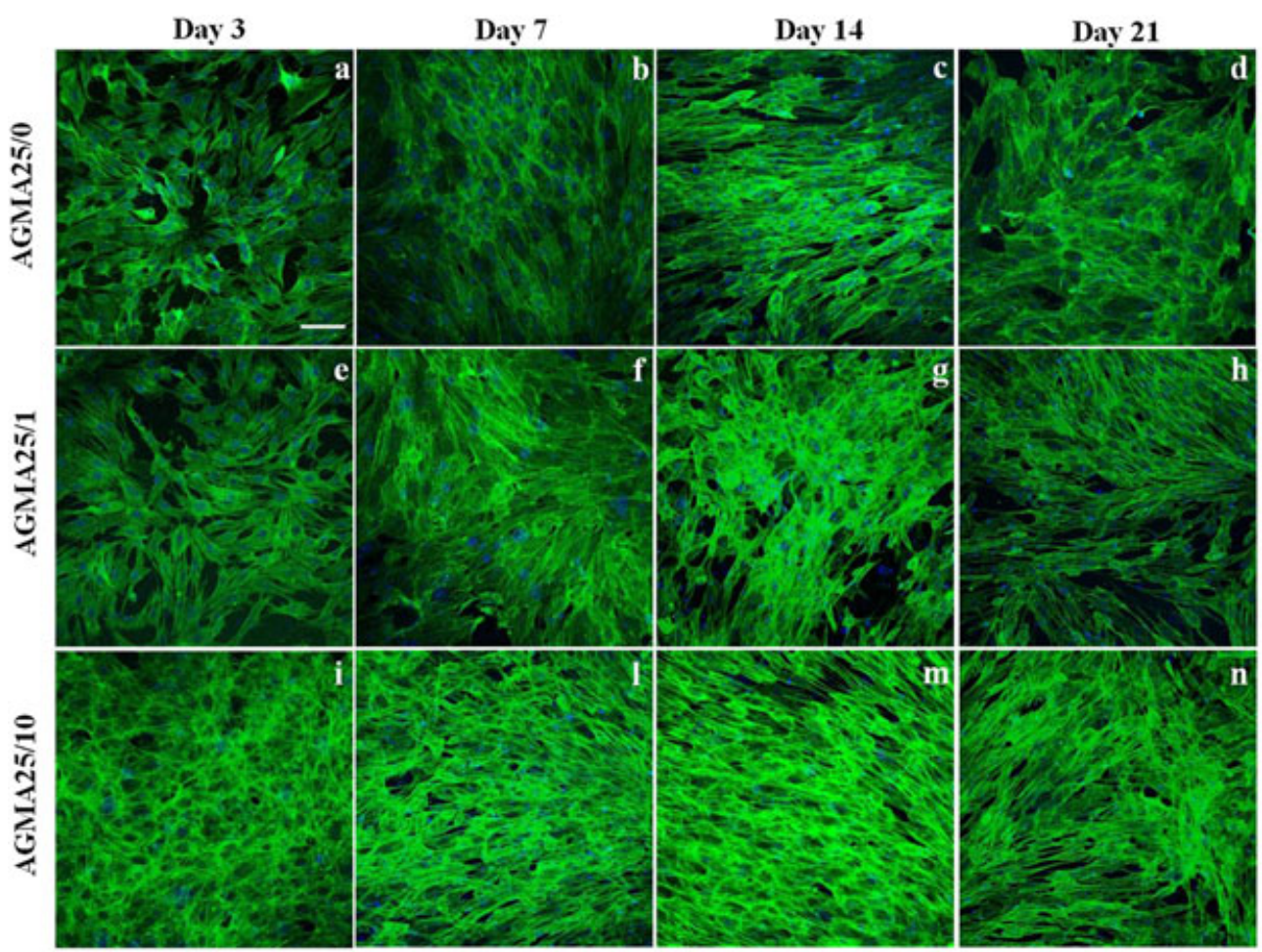

Figure 10. Confocal laser scanning micrographs of MC3T3-E1 cultured on AGMA25-MMT hydrogels; magnification $=\times 20$ (scale bar $=100 \mu \mathrm{m}$. 


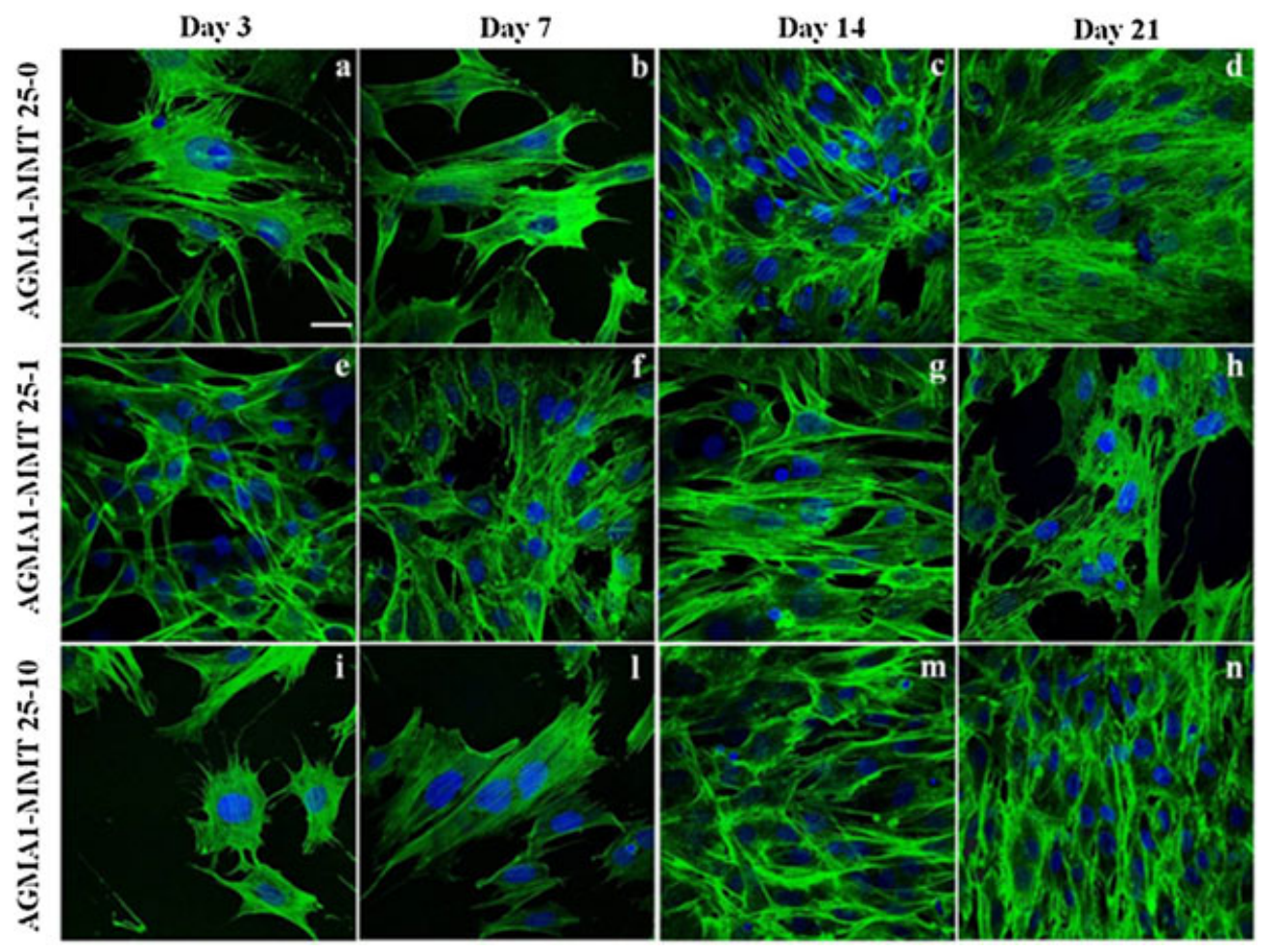

Figure 11. Confocal laser scanning micrographs of MC3T3-E1 cultured on AGMA25-MMT hydrogels; magnification $=\times 60$ (scale bar $=30 \mu \mathrm{m})$.]

associated with the expression of the main osteoblast functions (Figure 11).

\section{Conclusions}

In this study, the suitability of MMT reinforcement to improve the mechanical properties of RGD-like poly(amidoamine)-based hydrogels (AGMA1) was investigated, in order to render them suitable for osteoblast differentiation of MC3T3-E1 cells and for osteoblast culture. AGMA1 hydrogels responded favourably to MMT reinforcement whilst maintaining hydrophilicity and complete degradability in aqueous media at $\mathrm{pH}$ 7.4. Fully swollen AGMA1-MMT hydrogels showed $G^{\prime}$ values up to 20 times higher than those of common biomimetic hydrogels and of the same order or higher than the values reported for other hydrated materials proposed as scaffolds for osteoblast culture or osteogenic differentiation (Li et al., 2006; Dadsetan et al., 2012; Killion et al., 2013). This significant improvement was ascribed to the interpenetration between the polymer matrix and the inorganic filler, as suggested by the FT-IR, rheology and SANS studies. AGMA25-MMT hydrogels clearly induced differentiation of MC3T3-E1 cells towards the osteoblastic phenotype, as indicated by different osteoblast markers. Morphological analysis by CLSM confirmed these findings, showing an excellent cellular network throughout the hydrogel composite. These results underpin the conclusion that the combination of favourable properties of AGMA1-MMT hydrogel composites definitely warrants potential as scaffolds for osteoblast culture and bone grafts.

\section{Conflict of interest}

The authors have declared that there is no conflict of interest.

\section{Acknowledgements}

This project was funded by Cariplo Foundation, Italy (Grant No. 2010-501).

\section{References}

Aguzzi C, Cerezo P, Viseras C et al. 2007; Uses of clay minerals in semisolid health care and therapeutic products. Appl Clay Sci 36: 37-50.

Beck GR, Sullivan EC, Moran E et al. 1998; Relationship between alkaline phosphatase levels, osteopontin expression and mineralization in differentiating MC3T3-E1 osteoblasts. J Cell Biochem 68: 269-280.
Biswas M, Ray SS 2001; Recent progress in synthesis and evaluation of polymer-montmorillonite nanocomposites. Adv Polym Sci 155: 167-221. Cascone MG, Laus M, Ricci D et al. 1995. Evaluation of poly(vinyl alcohol) hydrogels as a component of hybrid artificial tissues. $J$ Mater Sci Mater Med 6: 71-75.

Chang CW, van Spreeuwel A, Zhang C et al. 2010; PEG/clay nanocomposite hydrogel: a mechanically robust tissue engineering scaffold. Soft Matter 6: 5157-5164.

Charles-Harris M, Koch MA, Navarro M et al. 2008; PLA/calcium phosphate degradable composite material for bone tissue engineering: an in vitro study. J Mater Sci Mater Med 19: 1503-1513.

Dadsetan M, Giuliani M, Wanivenhaus F et al. 2012; Incorporation of phosphate group 
modulates bone cell attachment and differentiation on oligo(polyethylene glycol) fumarate hydrogel. Acta Biomater 8: 1430-1439.

Dos Reis G, Fenili F, Gianfelice A et al. 2010; Direct microfabrication of topographical and chemical cues for the guided growth of neural cell networks on polyamidoamine hydrogels. Macromol Biosci 10: 842-852.

Emilitri E, Guizzardi F, Lenardi C et al. 2008; Novel poly(amidoamine)-based hydrogels as scaffolds for tissue engineering. Macromol Symp 266: 41-47.

Ferruti P, Bianchi S, Ranucci E et al. 2005a; Novel poly(amido-amine)-based hydrogels as scaffolds for tissue engineering. Macromol Biosci 5: 613-622.

Ferruti P, Bianchi S, Ranucci E et al. 2005b; Novel agmatine-containing poly(amidoamine) hydrogels as scaffolds tissue engineering. Biomacromolecules 6: 2229-2235.

Ferruti P 2013; Poly(amidoamine)s: past, present, and perspectives. J Polym Sci A Polym Chem 51: 2319-2352.

Forni F, Iannuccelli V, Coppi G et al. 1989; Effect of montmorillonite on drug release from polymeric matrices. Arch Pharm 322: 789-793.

Franchini J, Ranucci E, Ferruti P et al. 2006; Synthesis, physicochemical properties, and preliminary biological characterizations of a novel amphoteric agmatine-based poly (amidoamine) with RGD-like repeating units. Biomacromolecules 7: 1215-1222.

Giannelis EP 1998; Polymer-layered silicate nanocomposites: synthesis, properties and applications. Appl Organomet Chem 12: 675-680.

Haroun AA, Gamal-Eldeen A, Harding DRK 2009; Preparation, characterization and in vitro biological study of biomimetic three-dimensional gelatin-montmorillonite/cellulose scaffold for tissue engineering. J Mater Sci Mater Med 20: 2527-2540.

Huang FY, Wang YZ, Wang XL et al. 2005; Preparation and characterization of a novel biodegradable poly(p-dioxanone)/ montmorillonite nanocomposite. $J$ Polym Sci A Polym Chem 43: 2298-2303.

Hutmacher DW, Schantz JT, Lam CXF et al. 2007; State of the art and future directions of scaffold-based bone engineering from a biomaterials perspective. J Tissue Eng Regen M 1: 245-260.

Iyer P, Walker KJ, Madihally SV 2012; Increased matrix synthesis by fibroblasts with decreased proliferation on synthetic chitosangelatin porous structures. Biotechnol Bioeng 109: 1314-1325.
Jacchetti E, Emilitri E, Rodighiero S et al. 2008; Biomimetic poly(amidoamine) hydrogels as synthetic materials for cell culture. $J$ Nanobiotechnol 6: 1-14.

Joshi GV, Kevadiya BD, Mody HM et al. 2012; Confinement and controlled release of quinine on chitosan-montmorillonite bionanocomposites. J Polym Sci A Polym Chem 50: 423-430.

Kawasumi M 2004; The discovery of polymer-clay hybrids. J Polym Sci A Polym Chem 42: 819-824.

Killion JA, Kehoe S, Geever LM et al. 2013 Hydrogel/bioactive glass composites for bone regeneration applications: synthesis and characterisation. Mater Sci Eng C 33 4203-4212.

Lazzeri L, Barbani N, Cascone MG et al. 1994; Physicochemical and mechanical characterization of hydrogels of poly(vinyl alcohol) and hyaluronic acid. J Mater Sci Mater Med 5: 862-867.

Lee WF, Fu YT 2003; Effect of montmorillonite on the swelling behavior and drug-release behavior of nanocomposite hydrogels. J Appl Polym Sci 89: 3652-3660.

Li Q, Wang J, Shahani S et al. 2006; Biodegradable and photocrosslinkable polyphosphoester hydrogel. Biomaterials 27: 1027-1034.

Ma D, Xie X, Zhang ML 2009; Changes in the properties of a montmorillonite-water system during the adsorption and desorption of water: hysteresis. $J$ Polym Sci B Polym Phys 47: 740-749.

Madejovà J 2003; FTIR techniques in clay mineral studies. Vib Spectrosc 31: 1-10.

Magnaghi V, Conte V, Procacci P et al. 2011; Biological performance of a novel biodegradable polyamidoamine hydrogel as guide for peripheral nerve regeneration. $J$ Biomed Mater Res A 98: 19-30.

Martello F, Tocchio A, Tamplenizza M et al. 2014; Polyamidoamine-based hydrogels with tailored mechanical properties and degradation rates for tissue engineering. Acta Biomater 10: 1206-1215.

Mauro N, Manfredi A, Ranucci E et al. 2012; Degradable poly(amidoamine) hydrogels as scaffolds for in vitro culturing of peripheral nervous system cells. Macromol Biosci 13: 332-347.

Mujumdar SK, Siegel RA 2008; Introduction of pH-sensitivity into mechanically strong nanoclay composite hydrogels based on N-isopropylacrylamide. J Polym Sci A Polym Chem 46: 6630-6640.

Nishikawa T, Kokubu M, Kato Het al. 2012; Confocal laser scanning microscopy in study of bone calcification. Appl Surf Sci 262: 64-68.
Okay O, Oppermann W 2007; Polyacrylamideclay nanocomposite hydrogels: rheological and light scattering characterization. Macromolecules 40: 3378-3387.

Ozkan S, Kalyon DM, Yu X et al. 2009; Multifunctional protein-encapsulated polycaprolactone scaffolds: fabrication and in vitro assessment for tissue engineering. Biomaterials 30: 4336-4347.

Paluszkiewicz C, Stodolak E, Hasik M et al. 2011; FT-IR study of montmorillonite-chitosan nanocomposite materials. Spectrochim Acta A 79: 784-788.

Ranucci E, Ferruti P, Lattanzio E et al. 2009; Acid-base properties of poly(amidoamine)s. J Polym Sci A Polym Chem 4: 6977-6991.

Ray SS, Okamoto M 2003; Polymer/layered silicate nanocomposites: a review from preparation to processing. Prog Polym Sci 28: 1539-1641.

Renò F, Carniato F, Rizzi M et al. 2013; POSS/gelatin-polyglutamic acid hydroge composites: preparation, biological and mechanical characterization. J Appl Polym Sci 129: 699-706.

Richardson S, Ferruti P, Duncan R 1999; Polyamidoamines as potential endosomolytic polymers: evaluation in vitro and body distribution in normal and tumour-bearing animals. J Drug Targeting 6: 391-404.

Sellgren KL, Ma T 2012; Perfusion conditioning of hydroxyapatite-chitosan-gelatin scaffolds for bone tissue regeneration from human mesenchymal stem cells. J Tissue Eng Regen Med 6: 49-59.

Sharifi S, Blanquer SBG, van Kooten TG et al. 2012; Biodegradable nanocomposite hydrogel structures with enhanced mechanical properties prepared by photo-crosslinking solutions of poly(trimethylenecarbonate)poly(ethyleneglycol)-poly (trimethylenecarbonate) macromonomers and nanoclay particles. Acta Biomater 8: 4233-4243.

Söderqvist Lindblad M, Albertsson AC, Ranucci E et al. 2005; Biodegradable polymers from renewable sources: rheological characterization of hemicellulose-based hydrogels. Biomacromolecules 6: 684-690.

Stanford CM, Jacobson PA, Eanes ED et al. 1995; Rapidly forming apatitic mineral in an osteoblastic cell line. J Biol Chem 270: 9420-9428.

Tortelli F, Cancedda R 2009; Three-dimensional cultures of osteogenic and chondrogenic cells: a tissue engineering approach to mimic bone and cartilage in vitro. Eur Cells Mater 17: 1-14. 